\title{
Electromagnetic properties of polytetrafluoroethylene at microwave frequencies using finite element modeling waveguide adapter
}

\begin{abstract}
Experimental and theoretical approaches were shown great potential to determine electromagnetic properties of dielectric materials at microwave frequencies. In this study, the application of Finite Element Modeling (FEM) of waveguide adapter was utilized to investigate the distribution of electric and magnetic fields intensity of Polytetrafluoroethylene (PTFE) as dielectric sample. Essentially, the electric and magnetic fields intensity in various regions of waveguide were obtained. The computations of the reflection and transmission coefficients of dielectric sample were determined by implementation of Finite Element Methods and Nicolson-Rose-Wire (NRW) method as well. The results were compared with the experimental achievement results using the waveguide adapter in conjunction with a Vector Network Analyzer (VNA) at Microwave frequencies. The general observation indicate that, the level of transmission was greater than reflection for PTFE as dielectric material sample. Furthermore, among the two applied methods, the FEM is more accurate than the NRW method.
\end{abstract}

Keyword: Finite element method; Microwave measurement; PTFE; Waveguide adapter 